\title{
Single-Electron Transport in Electrically Tunable Nanomagnets
}

\author{
J. Fernández-Rossier ${ }^{1}$ and Ramón Aguado ${ }^{2}$ \\ ${ }^{1}$ Departamento de Física Aplicada, Universidad de Alicante, San Vicente del Raspeig, Spain \\ ${ }^{2}$ Instituto de Ciencia de Materiales de Madrid, CSIC, Cantoblanco, 28049 Madrid, Spain
}

(Received 19 April 2006; published 9 March 2007)

\begin{abstract}
We study a single-electron transistor (SET) based upon a II-VI semiconductor quantum dot doped with a single-Mn ion. We present evidence that this system behaves like a quantum nanomagnet whose total spin and magnetic anisotropy depend dramatically both on the number of carriers and their orbital nature. Thereby, the magnetic properties of the nanomagnet can be controlled electrically. Conversely, the electrical properties of this SET depend on the quantum state of the Mn spin, giving rise to spindependent charging energies and hysteresis in the Coulomb blockade oscillations of the linear conductance.
\end{abstract}

DOI: 10.1103/PhysRevLett.98.106805

Nanomagnets attract interest both because of their intriguing behavior as relatively macroscopic quantum objects and their potential technological applications as magnetic bits [1] and qbits [2]. The two fundamental properties of a nanomagnet are the net spin of its ground state, $S$ and its magnetic energy anisotropy tensor, $\mathcal{K}$ that governs the stability of the magnetization with respect to quantum and thermal fluctuations. Although recent experiments show that single-molecule magnets like $\mathrm{Mn}_{12}$ [3,4] or metallic Co [5] nanoparticles can be probed in singlemolecule transistor measurements, their properties can hardly be tuned once they are fabricated. Here we show that a single-electron transistor (SET) consisting of a II-VI quantum dot doped with a single-Mn atom behaves like a tunable nanomagnet whose magnetization and anisotropy axis can be reversibly manipulated electrically. Conversely, the conductance and charging energy of the tunable nanomagnet depend on the quantum state of the Mn spin and are not uniquely determined by the gate and the bias voltage.

Our proposal is based on two independent progress in nanofabrication. On one side, the fabrication and optical probing of single CdTe quantum dots doped with a single$M n$ atom [6-8]. In the absence of carriers, the spin $S=$ $5 / 2$ of the $\mathrm{Mn}$ is free. Optical excitation of electron-hole pairs into the dot shows that the Mn spin is exchange coupled to both the electron and the hole [6-9]. On the other side, the control of the charge state of II-VI semiconductor quantum dots with single-electron accuracy has been experimentally demonstrated $[10,11]$ as well as in the case of single-Mn-doped quantum dots [12] and Mn-doped GaAs islands [13].

Hamiltonian. - We consider a CdTe quantum dot (QD) doped with a single-Mn, weakly coupled to two metallic and nonmagnetic electrodes. The dot can be gated so that either the valence band or the conduction band is in resonance with the metallic reservoir and the number of either electrons or holes is varied at will. The total Hamiltonian reads $\mathcal{H}=\mathcal{H}_{\mathrm{QD}}+\mathcal{H}_{C}+\mathcal{H}_{L}+\mathcal{H}_{R}+\mathcal{V}_{L}+\mathcal{V}_{R}$. Here $\mathcal{H}_{\mathrm{QD}}$ is the Hamiltonian for the diluted magnetic
PACS numbers: 73.23.Hk, 78.55.Et, 78.67.Hc, 85.75.-d

semiconductor (DMS) quantum dot. In analogy with the standard model [14] for bulk DMS, $\mathcal{H}_{\mathrm{QD}}$ describes confined conduction-band electrons and valence holes interacting with a localized $\mathrm{Mn}$ spin $S=\frac{5}{2}$, denoted as $\vec{M}$, via a local exchange interaction. QD carriers occupy localized spin orbitals $\phi_{\alpha}$ with energy $\epsilon_{\alpha}$ which are described in the envelope function $\vec{k} \cdot \vec{p}$ approach $[9,15,16]$. In the case of valence band holes the 6 band KohnLuttinger Hamiltonian, including spin orbit interaction, is used as a starting point to build the quantum dot states [16]. The Hamiltonian of the isolated dot reads

$$
\mathcal{H}_{\mathrm{QD}}=\sum_{\alpha, \alpha^{\prime}}\left(\epsilon_{\alpha} \delta_{\alpha, \alpha^{\prime}}+J_{\alpha, \alpha^{\prime}} \vec{M} \cdot \vec{S}_{\alpha, \alpha^{\prime}}\right) f_{\alpha}^{\dagger} f_{\alpha^{\prime}}
$$

Here $f_{\alpha}^{\dagger}$ creates a band carrier in the $\alpha$ single-particle state of the quantum dot, which can be either a valence band or a conduction-band state. The first term in the Hamiltonian describes noninteracting carriers in the dot and the second term describes the exchange coupling of the carriers and the Mn. We neglect interband exchange so that $J_{\alpha, \alpha^{\prime}}=J_{e}$ $\left(J_{\alpha, \alpha^{\prime}}=J_{h}\right)$ if both $\alpha$ and $\alpha^{\prime}$ belong to the conductionband states (valence band states). In contrast, we include exchange processes by which a carrier is scattered between two different levels of the dot that belong to the same band. The matrix elements of both valence and conduction-band spin density, evaluated at the location of the Mn atom, are given by $\vec{S}_{\alpha, \alpha^{\prime}}$. They depend strongly on the orbital nature of the single-particle level in question. In the case of conduction band we neglect spin orbit interactions so that $\vec{S}_{\alpha, \alpha^{\prime}}$ is rotationally invariant [15]. In contrast, strong spin orbit interaction of the valence band makes the Mn-hole interaction strongly anisotropic $[9,12,16]$ and it varies between different dot levels. Following previous work $[9,15,16]$, confinement is described by a hard wall cubic potential with $L_{z}<L_{x} \simeq L_{y}$. Although real dots are not cubic, this simple model $[9,16]$ provides an excellent description of the Hamiltonian of the Mn spin coupled to the carriers, which is able to account for the nontrivial single- 
exciton photoluminescence (PL) spectra both for neutral [6-8] and charged [12] single-Mn-doped CdTe QD.

Coulomb repulsion between carriers is described within the constant interaction model: $\mathcal{H}_{C}=\frac{1}{2 C}\left(\hat{Q}+C_{g} V_{g}+\right.$ $\left.C_{L} \frac{\mu_{L}}{e}+C_{R} \frac{\mu_{R}}{e}\right)^{2}$, where $C=C_{L}+C_{R}+C_{g}$ is the total capacitance to the external circuit, $C_{L}$ and $C_{R}$ are the capacitances of the left and right junctions $\left(e V_{B} \equiv \mu_{L}-\mu_{R}\right.$ is the bias voltage) and $C_{g}$ is the capacitance to the gate (with voltage $V_{g}$ ). $\hat{Q}$ is the extra charge in the dot. We do not consider dots with orbital degeneracy for which Coulomb correlations, neglected in this Letter, are relevant [17,18]. Finally, $\mathcal{H}_{L}=\sum_{\sigma, k} \epsilon_{k} a_{k \sigma}^{\dagger} a_{k \sigma}$ and $\mathcal{H}_{R}=$ $\sum_{\sigma, p} \epsilon_{p} b_{p \sigma}^{\dagger} b_{p \sigma}$ describe the metallic electrodes and $\mathcal{V}_{L}=$ $\sum_{\sigma, k, \alpha} V_{\sigma, k, \alpha} f_{\alpha}^{\dagger} a_{k \sigma}+$ H.c. and $\mathcal{V}_{R}=\sum_{\sigma, p, \alpha} V_{\sigma, k, \alpha} f_{\alpha}^{\dagger} b_{p \sigma}+$ H.c. are the standard spin-conserving tunneling Hamiltonian that couple the metallic reservoirs and the dot.

We first discuss the properties of the eigenstates $|N\rangle$ of $\mathcal{H}_{\mathrm{QD}}$ for isolated dots $\left(\mathcal{V}_{L}=\mathcal{V}_{R}=0\right)$ with a given number of carriers, interacting with the $\mathrm{Mn}$ atom. We show results for two dots of CdTe with $L_{z}=60 \AA, L_{x}=$ $80 \AA$ and different $L_{y}=80 \AA$ (dot A) and $L_{y}=75 \AA$ (dot B), both doped with $1 \mathrm{Mn}$ atom. The neutral dot has 6 degenerate states, corresponding to the $(2 S+1)$ equivalent spin orientations of the $S=5 / 2 \mathrm{Mn}$ spin. This degeneracy is lifted in the presence of either electrons or holes. We focus on dots with a odd number of carriers (open shells) for which the interactions are stronger $[15,17]$ and study how the magnetic anisotropy varies with the number of carriers. The spectra of dots with 1 electron, 1 hole, and 3 holes are shown in Figs. 1(a)-1(c) respectively, for dot A (and also QD B for the case of 1 hole). The effect of intralevel exchange is magnified in the inset of Fig. 1(a). In Figs. 1(b) and 1(c) we only show the low-energy manifold for dot A with 1 and 3 holes. The 12 states of the low-

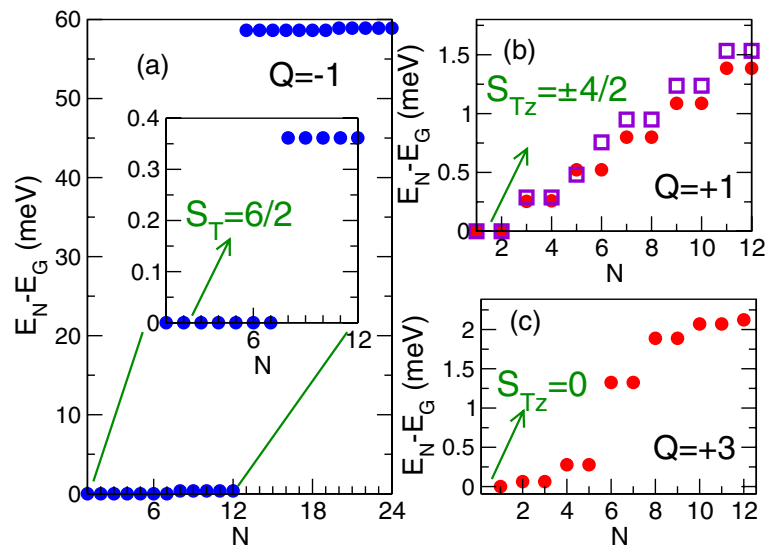

FIG. 1 (color online). Low-energy spectra of $\mathcal{H}_{\mathrm{QD}}$ for dot A (circles) with $Q=-1$ (a), $Q=+1$ (b), and $Q=+3$ (c). Notice the different vertical scale in (a) and (b) In panel (b) we also show the spectrum of QD B with $Q=+1$ (squares). The Kohn-Luttinger parameter for CdTe are $\gamma_{1}=4.14, \gamma_{2}=1.09$, and $\gamma_{3}=1.62$ and the spin orbit interaction is $\Delta=950 \mathrm{meV}$. We take $J_{e}=-15 \mathrm{eV} \AA^{3}$ and $J_{h}=60 \mathrm{eV} \AA^{3}$. energy manifold for $Q= \pm 1$ and $Q=+3$ are formed mainly by the two lowest-energy electronic configurations of the dot with a single unpaired fermion coupled to the 6 Mn states. The low-energy sector of $\mathcal{H}_{\mathrm{QD}}$ can be described by an intralevel effective Hamiltonian [12]: $\mathcal{H}_{\text {eff }}=j^{x} \tau^{x} M^{x}+j^{y} \tau^{y} M^{y}+j^{z} \tau^{z} M^{z}$, where $\tau^{a}$ are the Pauli matrices operating on the isospin space defined by the lowest-energy single-particle doublet.

Both the absolute and the relative values of $j_{x}, j_{y}$, and $j_{z}$ depend mostly on the spin properties of the external shell of the quantum dot: either conduction-band level $(Q=$ -1), heavy-hole $(Q=+1)$, or light-hole $(Q=+3)$. Thereby, the $j_{x, y, z}$ can be controlled reversibly by means of the gate voltage in the same device. The effective Hamiltonian of the Mn coupled to the "master fermion" in dot A goes from ferromagnetic Heisenberg $\left(j_{x}=j_{y}=\right.$ $\left.j_{z}<0\right)$ when $(Q=-1)$ to antiferromagnetic Ising $j_{x}=$ $j_{y}=0, j_{z}>0$ when $(Q=+1)$ to $X X Z\left(j_{x}=j_{y}>j_{z}\right)$. In dot B similar results are obtained, with $j_{x} \neq j_{y}$, which provides a spin-flip term in the $Q=+1$ case, absent in $\operatorname{dot} \mathrm{A}$.

Correspondingly, the spin properties of the ground state also change as a function $Q$. In the case of $Q=-1$ the $\mathrm{Mn}$ spin 5/2 and the conduction-band electron couple ferromagnetically to yield a septuplet with $S_{T}=3$. In the case of $Q=+1$ the ground state doublet corresponds to the $\mathrm{Mn}$ spin maximally polarized against the heavy-hole spin, $\left|M_{z}=-5 / 2, \uparrow\right\rangle$ and $\left|M_{z}=+5 / 2, \downarrow\right\rangle$, both for dots A and B. In dot A the rest of the low-energy sector is formed by 5 doublets eigenstates of both $M_{z}$ and $\tau^{z}$, whereas in dot B the small spin-flip interaction mixes $\left|M_{z}=+1 / 2, \downarrow\right\rangle$ and $\left|M_{z}=-1 / 2, \uparrow\right\rangle$. In the case with $Q=+3$, the ground state is not degenerate and the $\mathrm{Mn}$ spin is polarized in the $x y$ plane, minimizing $M_{z}$. These differences reflect the spin properties of conduction-band electron, heavy-hole and light-hole, respectively.

We now address how these remarkably different magnetic properties occurring in the same dot are reflected in the electrical behavior of the SET. In analogy with previous work [19-23], we derive a quantum master equation for the dissipative dynamics of the reduced density matrix $\rho_{\mathrm{NM}}(t)$ written in the basis of many-body states $|N\rangle$. Importantly, this quantum master equation includes the combined dynamics of both populations and coherences. The latter are important because of the intrinsic many-body degeneracies of the QD spectra shown in Fig. 1. Assuming that the quantum dot is weakly coupled to the electronic reservoirs (sequential tunneling), the dissipative dynamics of the density matrix is governed by a Markovian kernel, $\dot{\rho}(t)=A \rho(t)$, where $\rho$ can be casted as a vector containing both populations and coherence terms. The matrix $A$ contains information about dissipative dynamics of $\rho$ which is governed by the rates:

$$
\Gamma_{\bar{N}, M}^{ \pm}=\sum_{r \in L, R} \Gamma_{r} n_{r}^{ \pm}\left(E_{N}-E_{M}\right) \sum_{\alpha}\left|\left\langle N\left|f_{\alpha}^{ \pm}\right| M\right\rangle\right|^{2} .
$$


Here $f_{\alpha}^{+} \equiv f_{\alpha}^{\dagger}, f_{\alpha}^{-} \equiv f_{\alpha}, n_{r}^{+}$is the Fermi function of reservoir $r$ and $n_{r}^{-}=1-n_{r}^{+}$. The notation $\Gamma_{N, M}^{ \pm}$implies that states $M$ with charge $Q$ are connected with states $N$ with charge $Q \pm 1$. The coupling to the leads is parametrized by $\Gamma_{L, R}=\frac{2 \pi}{\hbar}\left|V_{L, R}\right|^{2} N_{L, R}$, where $N_{L, R}$ is the density of states of the metallic reservoir. Once we obtain the steady state density matrix $\tilde{\rho}$ (namely, $A \tilde{\rho}=0$ ), we can compute the average charge, magnetization and current. To lowest order in $\Gamma_{L, R}$, the most general expression for the current can be written as $I=\frac{I_{L}-I_{R}}{2}$ with

$$
\begin{aligned}
I_{L / R}= & e \Gamma_{L / R} \sum_{N, N^{\prime}} \sum_{M} \sum_{\alpha} \tilde{\rho}_{N, N^{\prime}}\left\{n_{L / R}^{+}\left(E_{N}-E_{N^{\prime}}\right)\right. \\
& \times\left\langle N^{\prime}\left|f_{\alpha}\right| M\right\rangle\left\langle M\left|f_{\alpha}^{\dagger}\right| N\right\rangle \\
& \left.-n_{L / R}^{-}\left(E_{N}-E_{M}\right)\left\langle N^{\prime}\left|f_{\alpha}^{\dagger}\right| M\right\rangle\left\langle M\left|f_{\alpha}\right| N\right\rangle\right\} .
\end{aligned}
$$

Notice that Eq. (3) includes both diagonal and nondiagonal terms in the density matrix. The latter are important when two degenerate states with $Q$ are coupled to the same state of $Q \pm 1$ via a single tunneling event.

The steady state of a standard SET is uniquely characterized by external voltages. For instance, a new charge is accommodated in the dot at precise values of the gate voltage, when the electrochemical potential of the dot $\mu(N)$ (the energy required for adding the $N$ th electron to the dot) falls within the bias window $\mu_{L} \geq \mu(N) \geq \mu_{R}$. When this condition is met, the number of electrons can vary between $N-1$ and $N$ resulting in a single-electron tunneling current. Importantly, in our case the charge and the conductance of the SET depend also on the quantum state of the Mn spin.

In Fig. 2 we show linear conductance $G_{0}\left(V_{G}\right)$, average charge and diagonal terms of the steady state $\rho$, as the gate produces the transition between charge zero and charge \pm 1 for both electrons (left panels) and holes (right panels). The
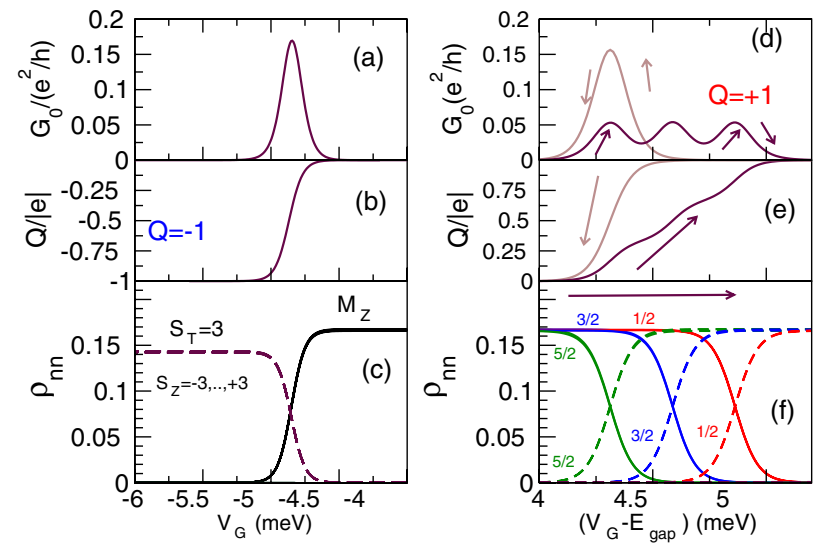

FIG. 2 (color online). $\quad G_{0}\left(V_{G}\right)$ (upper panels), charge (middle panels), and diagonal terms of the $\rho$ (lower panels) for QD A as a function of $V_{G}$ around the $Q=-1 \leftrightarrow 0$ transition (left) and $Q=0 \leftrightarrow+1$ (right). Lower panels: solid (dashed) lines correspond to $\mathcal{Q}=0(|\mathcal{Q}|=1)$ states. Results obtained with $\Gamma_{L}=$ $\Gamma_{R}=0.01 \mathrm{meV}$ and $k_{B} T=0.05 \mathrm{meV}$. initial $V_{G}$ for the charging simulations is chosen so that only the $\mathcal{Q}=0$ states are occupied. This initial condition is described by a thermal $\rho$ with 6 equally populated $\mathrm{Mn}$ spin states, $M_{z}= \pm 5 / 2, \pm 3 / 2, \pm 1 / 2$. We ramp the gate and solve the master equation to obtain the steady state $\rho$, which is used as initial condition for the next run with higher $V_{G}$. In the case of electrons we obtain standard results: a single peak in the $G_{0}\left(V_{G}\right)$ curve occurs as the gate is ramped to change the charge of the dot by one unit. In Fig. 2(c) we show the evolution of the steady state populations: The $6 \mathcal{Q}=0$ spin states are relaxed altogether in favor of the 7 states of the $Q=1$ and $S_{T}=3$ states.

The results for holes in QD A are remarkably different: in the process of injection of 1 hole the $G_{0}\left(V_{G}\right)$ curve shows 3 peaks instead of 1 . This results from the lack of Mn spin relaxation $\left(M_{z}\right.$ is a conserved quantity for the entire Hamiltonian including tunneling), which makes the steady $\rho$ different from to the thermal $\rho$. As the gate brings the two $\mathcal{Q}=+1$ ground states $(+5 / 2, \downarrow)$ and $(-5 / 2, \uparrow)$ in resonance with the six ground states with $Q=0$, population tranfer only affects states with $\left|M_{z}\right|=5 / 2$ in both charge sectors [first peak in Fig. 2(d) at $V_{G}=4.3 \mathrm{meV}$ ]. Further increase of the gate brings the energy of the $Q=$ +1 ground state doublet below the $Q=0$ states with $\left|M_{z}\right| \neq 5 / 2$, which are not depleted because $M_{z}$ is conserved [Fig. 2(f)]. The population transfer only occurs when the second and third doublet of the $Q=+1$ spectrum, with $\left|M_{z}\right|=3 / 2$ and $\left|M_{z}\right|=1 / 2$ are brought in resonance with the $Q=0$ states. This accounts for the other two peaks in the $G_{0}\left(V_{G}\right)$ curve as the charge of the dot approaches +1 . Hence, the charging energy for holes depends on the absolute value of the spin of the Mn. The discharge simulation is done analogously. If the initial $V_{G}$ is such that there is one hole in the QD, the Ising interaction removes the degeneracy among states with different $\left|M_{z}\right|$. Only the doubly degenerate ground state of the $\mathcal{Q}=+1$ sector is occupied in thermal equilibrium. As the gate is ramped to discharge the dot, a single peak in the conductance is obtained, corresponding to the resonance condition with between the $\mathcal{Q}=0$ and $\mathcal{Q}=+1$ states with $\left|M_{z}\right|=5 / 2$.

The difference between electrons and holes arises from the different value of a crucial time scale in the magnetic single electron transistor: the Mn spin relaxation time, $T_{1}$. In the case of QD A, $T_{1}$ is infinite for holes (Ising coupling) which makes the steady state different from the thermal state. In the case of electrons (left panels) the transverse spin interactions make $T_{1}$ comparable to the charge relaxation time $\left(\Gamma_{L, R}^{-1}\right)$ so that steady and thermal $\rho$ are identical. In real dots doped with one hole, $T_{1}$ may be long but not infinite. Two independent mechanisms, missing in the simulations shown in the right panels of Fig. 2, yield a finite $T_{1}$. First, the $\mathrm{Mn} T_{1}$ due to superexchange with other Mn spins which scales exponentially with the Mn density [24]. For bulk $\mathrm{Cd}_{0.995} \mathrm{Mn}_{0.005} \mathrm{Te}$ we have $T_{1}=10^{-3} \mathrm{~s}$, 

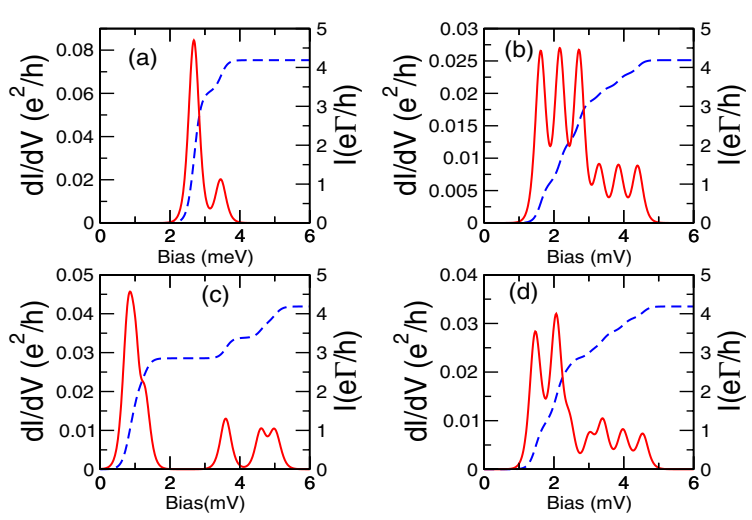

FIG. 3 (color online). Current and differential conductance as a function of bias for QD A (a),(b),(c) at various charge states and QD B (d) (see text).

which is a lower limit estimate for $T_{1}$ of the QD with a single Mn. The second mechanism is the small [12] transverse spin interaction, which is proportional to the lighthole heavy-hole mixing. We have simulated QD B, for which spin-flip interaction between the hole and the Mn is small but nonzero resulting in a finite $T_{1}$. If we integrate the master equation for $\Gamma^{-1} \ll t \ll T_{1}$ the $G_{0}\left(V_{G}\right)$ curve displays 2 peaks and hysteretic behavior. In contrast, if we integrate the master equation for $\Gamma^{-1} \ll T_{1} \ll t$, the system reaches the equilibrium state for each value of $V_{G}$ so that the $G_{0}\left(V_{G}\right)$ curve has a single peak. Therefore, we claim that effects related to incomplete spin relaxation of the dot will be observed subject to two conditions: the finite bandwith of the measurements should be larger than $1 / T_{1}$ (see, for instance, Ref. [4]) and the pace at which $V_{G}$ is ramped should be faster than $T_{1}$.

The finite bias conductance of the device also depends strongly on the charge state of the dot. In Fig. 3 we show $I\left(V_{B}\right)$ and $\frac{d I}{d V_{B}}$ curves for dot A corresponding to biasassisted single-electron fluctuations between $Q=0$ and $Q=-1$ [Fig. 3(a)], $Q=0$ and $Q=+1$ [Fig. 3(b)], and $Q=2$ and $Q=+3$ [Fig. 3(c)]. Figure 3(d) is the analogous of $3 \mathrm{~b}$ for dot $\mathrm{B}$. Current flows whenever the addition of a fermion is permitted by energy conservation and spin selection rules. The former provides a link between the $\frac{d I}{d V_{B}}$ curve and the energy spectra of dots shown in Fig. 1 (since the spectra of dots with $Q=0$ and $Q=2$ are flat). Interestingly, the $\frac{d I}{d V_{B}}$ for electron tunneling [3(a)] shows a zero-magnetic field splitting related to recent experimental observations [25].

In summary, we have shown some of the equilibrium and nonequilibrium properties of a semiconductor quantum dot doped with a single-Mn atom and wired as a singleelectron transistor. The different orbital nature of the conduction-band electrons, heavy holes, and light holes determines both the total spin and the magnetic anisotropy of the dot. In the case of holes, for which Mn spin-flip processes are heavily inhibited, we predict different results for the $G_{0}\left(V_{G}\right)$ curves depending on whether the system is relaxed to equilibrium or not. In the case of the latter, we predict hysteretic Coulomb blockade oscillations related to the quantum state of the Mn spin. Because most of the transport properties discussed above are inherent to nanomagnets with long spin relaxation time, our findings might be very general and have implications in recent experiments $[3,4,13]$.

Fruitful discussions with L. Brey, J. J. Palacios, Y. Léger, L. Besombes, J. Cibert, H. Mariette, C. Gould, and P. Hawrylak are acknowledged. This work has been financially supported by MEC-Spain (Grants No. FIS200402356, No. MAT2005-07369-C03-03, and the Ramon y Cajal Program) and by CAV (No. GV05-152).

[1] M. Jamet et al., Phys. Rev. Lett. 86, 4676 (2001).

[2] M. Leuenberger and D. Loss, Nature (London) 410, 789 (2001).

[3] H. B. Heersche et al., Phys. Rev. Lett. 96, 206801 (2006).

[4] Moon-Ho Jo et al., Nano Lett. 6, 2014 (2006).

[5] M. Deshmukh and D. Ralph, Phys. Rev. Lett. 89, 266803 (2002).

[6] L. Besombes et al., Phys. Rev. Lett. 93, 207403 (2004).

[7] L. Besombes et al., Phys. Rev. B 71, 161307 (2005).

[8] Y. Léger et al., Phys. Rev. Lett. 95, 047403 (2005).

[9] J. Fernández-Rossier, Phys. Rev. B 73, 045301 (2006).

[10] D. L. Klein et al., Nature (London) 389, 699 (1997).

[11] J. Seufert et al., Appl. Phys. Lett. 82, 3946 (2003).

[12] Y. Léger et al., Phys. Rev. Lett. 97107401 (2006).

[13] J. Wunderlich, Phys. Rev. Lett. 97, 077201 (2006).

[14] J. K. Furdyna, J. Appl. Phys. 64, R29 (1988).

[15] J. Fernández-Rossier and L. Brey, Phys. Rev. Lett. 93, 117201 (2004).

[16] F. V. Kyrychenko and J. Kossut, Phys. Rev. B 70, 205317 (2004).

[17] A. O. Govorov, Phys. Rev. B 72, 075359 (2005); A. O. Govorov, Phys. Rev. B 72, 075358 (2005).

[18] F. Qu and P. Hawrylak, Phys. Rev. Lett. 95, 217206 (2005).

[19] A. Efros, E. Rashba, and M. Rosen, Phys. Rev. Lett. 87, 206601 (2001).

[20] X. Waintal and P. Brouwer, Phys. Rev. Lett. 91, 247201 (2003).

[21] X. Waintal and O. Parcollet, Phys. Rev. Lett. 94, 247206 (2005).

[22] C. Timm and F. Elste Phys. Rev. B 73, 235304 (2006).

[23] M. Braun, J. König, and J. Martinek, Phys. Rev. B 70, 195345 (2004).

[24] J. Lambe and C. Kikuchi, Phys. Rev. 119, 1256 (1960); D. Scalbert et al., Solid State Commun. 66, 571 (1988).

[25] C. Gould et al., Phys. Rev. Lett. 97, 017202 (2006). 